\title{
Speed factors computed for pumping schedules in Water Distribution Networks: DDA versus PDA formulations
}

\author{
Remus Alexandru Mădulărea ${ }^{1}$, Petre-Ovidiu Ciuc $^{1}$, Andrei-Mugur Georgescu ${ }^{2}$, and Sanda-Carmen Georgescu ${ }^{1, *}$ \\ ${ }^{1}$ University POLITEHNICA of Bucharest, Power Engineering Faculty, 313 Spl. Independentei, 060042, Bucharest, Romania \\ ${ }^{2}$ Technical University of Civil Engineering Bucharest, Hydraulics and Environmental Protection Department, 124 Lacul Tei, \\ 020396, Bucharest, Romania
}

\begin{abstract}
This paper focuses on a methodology allowing to derive the pumping schedule in Water Distribution Networks (WDN), upon a time dependent water demand. The selected test case is a previously studied WDN. Two pumping algorithms give different pumping rules. By solving the nonlinear system of equations, consisting of energy balance equations, mass balance equations and pumping rules, one gets the pumps speed factors. Solutions attached to the Pressure Driven Analysis (PDA) correspond to energy and cost savings, with respect to the solutions given by the Demand Driven Analysis (DDA). The methodology described in this paper is simple and rapid, but the iterative numerical method used to solve the system of equations is highly dependent on the starting guess.
\end{abstract}

\section{Introduction}

Pumping schedules attached to variable speed driven pumps in Water Distribution Networks (WDN) are a major concern for water companies, because appropriate/ personalized schedules allow reducing significantly the overall energy consumption $[1,2]$.

Hydraulic analysis of WDNs can be performed using either specialised software (e.g. EPANET [3]; Bentley WaterGEMS; WDNetXL [4]; WaterNetGen [5] etc), or individually developed codes (e.g. built in GNU Octave $[6,7]$, MATLAB or other programming languages).

With respect to nodal demands [8], the hydraulic analysis can be mainly performed as Demand Driven Analysis - DDA (where outflows are pressure independent, being set as fixed boundary conditions at the consumers), or as Pressure Driven Analysis - PDA (where outflows are pressure dependent boundary conditions, varying upon the available pressure at each consumer $[9,10])$. Hybrid simulation methods combining DDA and PDA also can be used [6].

Among the above listed specialised software, only EPANET lacks the PDA formulation, but it incorporates emitters, which allow modelling pressure-dependent outflows at the consumers [11].

All listed software allow modelling variable speed driven pumps, through their speed factor $\omega$ (or relative speed), defined as ratio between the actual pump speed and its nominal speed. Speed factor values can be inserted into the model as fixed values over time, or through time dependent speed patterns [3] issued from computations performed outside the current software $[1,12]$, or through rule-based controls [3], e.g. where pump's speed factor is adjusted in imposed steps upon the pressure level available at a certain node [2, 13]. Within specialised software, speed factor values are not computed as solver outputs, excepting WDNetXL.

In this paper, we present a methodology to derive the pumping schedule in WDNs (to compute pumps speed factors), for both formulations: DDA and PDA. The selected test case is a simple WDN fed by variable speed driven pumps, previously studied [12]. PDA and DDA results are finally compared. DDA results are also discussed with respect to similar results obtained by Georgescu [12], using another DDA-based methodology.

\section{Speed factors computing method}

In order to simplify the presentation, the proposed methodology is developed for a test case, consisting of the WDN from figure 1, proposed in an open access paper by Georgescu [12]. The main components are tagged with ID labels, as in figure 1 , with ID $=1 \div 18$ for nodes (where ID $=1$ denotes the water source - the reservoir R), ID $=1 \div 20$ for pipes and ID $=21 \div 23$ for three variable speed driven pumps. The pumping station equipped with those pumps is bordered by the main suction node with ID $=2$, and the main discharge node with ID $=3$. There are 5 consumers (end-users) within the studied looped network, labelled with ID $\in\{11 ; 12$; $14 ; 16 ; 18\}$ and marked by red dots in figure 1 .

The hydraulic analysis is an Extended Period Simulation (EPS) analysis [14], conducted over a period of 24 hours, with a time step of one hour, starting at midnight; the time $t$ is the starting moment of each time step (thus, $t=1$ starts at mid-night and $t=24$ at $11 \mathrm{p} . \mathrm{m}$.). During a time step interval, the system behaviour fits a steady-state regime.

\footnotetext{
* Corresponding author: carmen.georgescu@upb.ro
} 


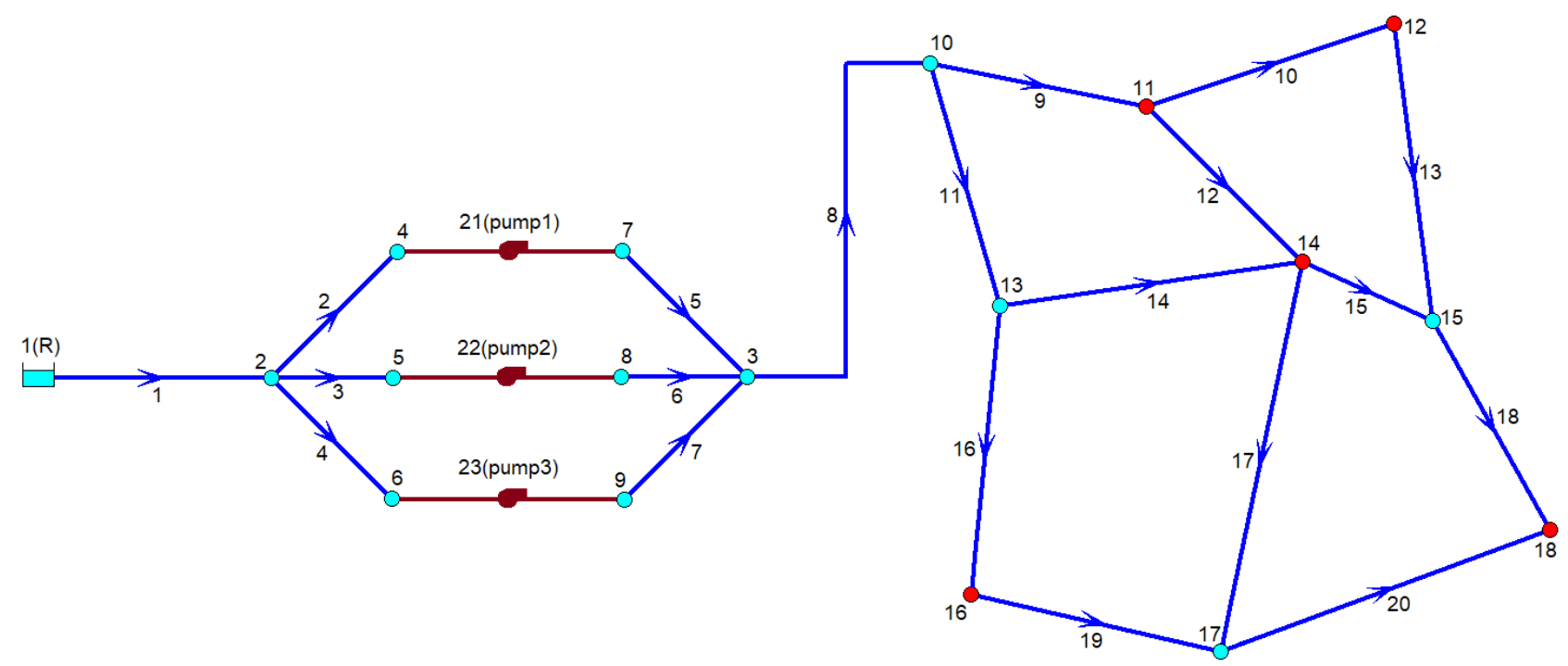

Fig. 1. WDN map with components ID labels: nodes from 1 to 18 (where 1 is the reservoir R), pipes from 1 to 20 , three pumps from 21 to 23; five nodes labelled as 11, 12,14, 16 and 18 (red dots) refer to consumers.

The EPS model evaluates successively all 24 steadystate intervals, considering the changes from one time step to the next one, through time dependent boundary conditions (here, the requested water demands).

Pipes geometry (diameter $D$, length $L$ and equivalent roughness), the water demand pattern coefficients $c_{q}(t)$ and the energy price pattern coefficients $c_{e}(t)$, dependent on time $t$ over the above defined 24 hours period, are fully described in Georgescu [12].

Each consumer requests a daily mean water demand $Q_{r m}$ of $0.088 \mathrm{~m}^{3} / \mathrm{s}$, so the requested hourly demand is computed as: $Q_{r}(t)=c_{q}(t) Q_{r m}$. The hourly energy price $C_{h}(t)$ is computed by multiplying the coefficients $c_{e}(t)$ with a daily mean price of $0.065 € / \mathrm{kWh}[15]$.

The hydraulic system is flat, e.g. with nodes elevation $z=0$. The reservoir's free surface is open to the atmosphere, so there the head is $H_{\mathrm{R}}=0$. The reference gauge pressure $p_{r e f}$, requested at each consumer to ensure the demand, is $p_{r e f}=40$ meters of water column (i.e. reference pressure head $H_{r e f}=40 \mathrm{~m}$ ). The minimal gauge pressure $p_{\min }$, for which there is outflow at the consumer, is set here as $p_{\min }=0 \mathrm{mWC}$ (i.e. minimal pressure head $H_{\text {min }}=0 \mathrm{~m}$ ); usually, $p_{\min }=2 \div 3 \mathrm{mWC}$.

The Darcy-Weisbach formula is used to compute head losses $h$ (in meters) on pipes:

$$
h=R \cdot Q^{2}=\frac{8 \lambda L}{g \pi^{2} D^{5}} Q^{2}
$$

where $Q$ is the flow rate (in $\mathrm{m}^{3} / \mathrm{s}$ ), $R$ is the hydraulic resistance (in $\left.\mathrm{s}^{2} / \mathrm{m}^{5}\right), g$ is the gravity $\left(g=9.81 \mathrm{~m} / \mathrm{s}^{2}\right)$, and $\lambda$ is the friction factor, defined by the Swamee and Jain formula $[3,6]$. Inlet and outlet kinetic terms on pipes, and minor losses are neglected.

At each moment $t$ (where $t=1 \div 24$, with hourly time step), the centrifugal pumps (denoted by indexes $i$ corresponding to ID-s from figure 1) operate at a certain speed $\omega_{i}(t)$ upon the following characteristic curves [12], namely the head - flow rate curve, with pumping head $H$ in meters, for the flow rate $Q$ in $\mathrm{m}^{3} / \mathrm{s}$ :

$$
H_{i}(t)=83\left(\omega_{i}(t)\right)^{2}-741.6\left(Q_{i}(t)\right)^{2} ; i=21 \div 23
$$

and the efficiency curve, with efficiency values $\eta$ in percents, for $Q$ in $\mathrm{m}^{3} / \mathrm{s}$ :

$$
\eta_{i}(t)=775\left(\frac{Q_{i}(t)}{\omega_{i}(t)}\right)-2250\left(\frac{Q_{i}(t)}{\omega_{i}(t)}\right)^{2} ; i=21 \div 23
$$

Within DDA, the available demand $Q_{a}(t)$ at each consumer equals the requested demand $Q_{r}(t)$ at each time step. Within the PDA formulation, $Q_{a}(t)$ is computed with the following time and pressure dependent demand relationship [13]:

$$
Q_{a}(t)=Q_{r}(t) \times\left\{\begin{array}{cl}
1 & p(t) \geq p_{\text {ref }} \\
\sqrt{\frac{p(t)-p_{\text {min }}}{p_{\text {ref }}-p_{\text {min }}} ;} & p_{\text {min }} \leq p(t)<p_{\text {ref }} \\
0 & p(t)<p_{\text {min }}
\end{array}\right.
$$

where $p(t)$, in $\mathrm{mWC}$, is the available pressure at the consumer, at time $t$.

For the studied WDN fed by 3 working pumps, the nonlinear system of equations, consisting of energy balance equations on all 23 links and continuity equations in 17 nodes (with ID from 2 to 18), yields a total of 40 equations with 43 unknowns, namely: 23 flow rate values $\left(Q_{1}\right.$ to $\left.Q_{23}\right), 17$ nodal head values $\left(H_{2}\right.$ to $H_{18}$, more precisely pressure head values for that flat system) and 3 speed factor values $\left(\omega_{21}, \omega_{22}\right.$ and $\left.\omega_{23}\right)$. The resulting underdetermined system of equations corresponds to a stiff problem.

Nonlinear system of equations can be solved in GNU Octave [16] or MATLAB, using the $f$ solve function [17], developed for minimization and zero finding through algorithms based on model-trust region optimization approach [18]; according to fsolve documentation [17], the system of equations cannot be underdetermined. So, the studied hydraulic problem needs 3 additional equations. 
One can add 2 equations related to speed factors settings, depending on the pumping station operating algorithm. Two options will be considered further $[6$, 12], namely:

(1) a classical pumping algorithm, where at a time $t$, a single pump runs at variable speed with $\omega(t) \neq 1$, while any other opened pump runs at its nominal speed, where the speed factor equals 1 .

(2) an improved pumping algorithm, where at a time $t$, all opened pumps run with equal speed factor values.

The missing third equation, needed to obtain a system of 43 equations with 43 unknowns, can be the global continuity equation, where the inflow $Q_{1}$ is the sum of the five available outflows, defined by (4):

$$
Q_{1}(t)=\sum_{j} Q_{a_{j}}(t) ; j \in\{11 ; 12 ; 14 ; 16 ; 18\}
$$

The global mass balance equation (5) is not linearly independent with respect to the other 17 continuity equations, but it helps to mimic a determined system of equations, which can be solved iteratively using the fsolve function, starting from an initial guess of the solution. As we will show further within Section 3, that pseudo-determined nonlinear system of equations is highly dependent on the starting guess: the algorithm converges only for a certain initial guess, while it fails for a slightly changed initial guess. The same hydraulic problem was solved in Georgescu [12], within a twosteps methodology and a DDA formulation: there, the fsolve function was used to solve a properly-determined nonlinear system (with 6 equations and 6 unknowns), and the same algorithm proved to be globally convergent.

The nonlinear system of equations derived in this paper is too long to be written explicitly here, but it can be presented conveniently as below, mentioning that the first 23 equations $\left(f_{1} \div f_{23}\right)$ are energy balance equations on links (on pipes with ID $=1 \div 4$, on pumps with ID = $21 \div 23$, then on pipes with ID $=5 \div 20$ ); the next 17 equations $\left(f_{24} \div f_{40}\right)$ are continuity equations in nodes $2 \div 18$; equation $f_{41}$ results from (5); the last 2 equations refer to speed factors (which differ for the classic pumping algorithm, with respect to the improved algorithm); in order to simplify notations, the time dependency was not mentioned here:

$$
\begin{aligned}
& f_{1}=H_{\mathrm{R}}-H_{2}-R_{1} Q_{1}\left|Q_{1}\right|=0 \\
& f_{2}=H_{2}-H_{4}-R_{2} Q_{2}\left|Q_{2}\right|=0 \\
& \quad \vdots \\
& f_{5}=H_{4}-83 \omega_{21}^{2}-741.6 Q_{21}^{2}-H_{7}=0 \\
& \quad \vdots \\
& f_{24}=Q_{1}-Q_{2}-Q_{3}-Q_{4}=0 \\
& \vdots \\
& f_{40}=Q_{18}-Q_{20}-Q_{a_{18}}=0 \\
& f_{41}=Q_{1}-Q_{a_{11}}-Q_{a}-Q_{a}-Q_{a_{16}}-Q_{a_{18}}=0 \\
& f_{42}=\omega_{22}-1=\left.0\right|_{\text {classic }} ; f_{42}=\omega_{22}-\omega_{21}=\left.0\right|_{\text {improved }} \\
& f_{43}=\omega_{23}-1=\left.0\right|_{\text {classic }} ; f_{43}=\omega_{23}-\omega_{21}=\left.0\right|_{\text {improved }}
\end{aligned}
$$

For the considered pumping station, we adopt further the following pumping rules [12]:

- rule no. 1: for $c_{q}(t) \leq 0.5$ (off-peak hours), where $t \in$ $\{1 \div 5 ; 23 ; 24\}$, pump1 is opened $\left(\omega_{21} \neq 0 ; Q_{21} \neq 0\right)$, while pump2 and pump3 are closed $\left(\omega_{22}=\omega_{23}=0\right.$; $Q_{22}=Q_{23}=0$ );

- rule no. 2: for $0.5<c_{q}(t)<1$, where $t \in\{6 ; 21 ; 22\}$, pump1 and pump2 are opened, while pump3 is closed $\left(\omega_{23}=0 ; Q_{23}=0\right) ;$ here, the classical pumping algorithm gives $\omega_{21} \neq 0 ; \omega_{22}=1 ; Q_{21} \neq 0 ; Q_{22} \neq 0$, while the improved algorithm gives $\omega_{21}=\omega_{22} \neq 0$; $Q_{21}=Q_{22} \neq 0$;

- rule no. 3: for $c_{q}(t)=1$ (attached to the daily mean regime, used in pumps selection $[6,12])$, where $t=7$, pump1 and pump2 operate at nominal speed $\left(\omega_{21}=\omega_{22}=1 ; Q_{21}=Q_{22} \neq 0\right)$, while pump3 is closed $\left(\omega_{23}=0 ; Q_{23}=0\right)$;

- rule no. 4: for $c_{q}(t)>1$ (peak hours), where $t=8 \div 20$, all 3 pumps are opened; here, the classical pumping algorithm gives $\omega_{21} \neq 0 ; \quad \omega_{22}=\omega_{23}=1 ; \quad Q_{21} \neq 0 ;$ $Q_{22}=Q_{23} \neq 0$, while the improved algorithm gives $\omega_{21}=\omega_{22}=\omega_{23} \neq 0 ; Q_{21}=Q_{22}=Q_{23} \neq 0$.

Within the system (6), the list of unknowns contains 3 type of variables: flow rates, nodal heads and speed factors; as already mentioned, $H_{\mathrm{R}}=0$. To obtain a compact form of the system, to be solved numerically, the unknowns become the components $w_{k}$ (with $k=$ $1 \div 43$ ) of a column vector $\mathbf{w}$, as:

$$
\begin{aligned}
& w_{1}, w_{2}, \cdots, w_{23} \equiv Q_{1}, Q_{2}, \cdots, Q_{23} \\
& w_{24}, w_{25}, \cdots, w_{40} \equiv H_{2}, H_{3}, \cdots, H_{18} \\
& w_{41}, w_{42}, w_{43} \equiv \omega_{21}, \omega_{22}, \omega_{23}
\end{aligned}
$$

Thus, the final nonlinear system of equations can be defined as $\mathbf{f}(\mathbf{w})=\mathbf{0}$, where the column vector $\mathbf{f}$ contains 43 components, namely the functions $f_{k}\left(w_{1}, w_{2}, \ldots, w_{43}\right)=0$, with $k=1 \div 43$. Taking into account (4), with reference pressure head $H_{r e f}=40 \mathrm{~m}$, and minimal pressure head $H_{\text {min }}=0$, the system (6) can be rewritten as:

$$
\begin{aligned}
& f_{1}=-w_{24}-R_{1} w_{1}\left|w_{1}\right|=0 \\
& f_{2}=w_{24}-w_{26}-R_{2} w_{2}\left|w_{2}\right|=0 \\
& \vdots \\
& f_{5}=w_{26}-83 w_{41}^{2}-741.6 w_{21}^{2}-w_{29}=0 \\
& \vdots \\
& f_{24}=w_{1}-w_{2}-w_{3}-w_{4}=0 \\
& \vdots \\
& f_{40}=w_{18}-w_{20}-Q_{r} \sqrt{w_{40} / H_{r e f}}=0, \text { if } w_{40}<H_{r e f} \\
& f_{40}=w_{18}-w_{20}-Q_{r}=0, \text { if } w_{40} \geq H_{r e f} \\
& \vdots \\
& f_{43}=w_{43}-1=\left.0\right|_{\text {classic }} ; f_{43}=w_{43}-w_{41}=\left.0\right|_{\text {improved }}
\end{aligned}
$$


Obviously, the solution of the system (8) corresponds to the pumping rule no. 4 . The system (8) simplifies to:

- 37 equations with 37 unknowns for the pumping rules no. 2 and 3 ;

- 31 equations with 31 unknowns for pumping rule no. 1 .

Finally, based on the parameters attached to the duty points of each pump: flow rate $Q_{i}(t)$, pumping head $H_{i}(t)$, and pump efficiency $\eta_{i}(t)$ defined by (3) in percents, the power $P_{i}(t)$ of each pump can be computed (in watts) at every time step, as:

$$
P_{i}(t)=\frac{\rho g Q_{i}(t) H_{i}(t)}{\eta_{i}(t) / 100} ; \quad i=21 \div 23
$$

where $\rho$ is the water density $\left(\rho=1000 \mathrm{~kg} / \mathrm{m}^{3}\right)$.

Starting from (9) with $t=1 \div 24$, one can compute the hourly energy consumption $E_{i}(t)$ for each pump, as well as the daily energy (denoted $E$ ) consumed by the pumping station. Considering the mentioned hourly energy price $C_{h}(t)$, one can compute, finally, the total $\operatorname{cost} C$ of the daily consumed energy.

\section{Results and discussions}

For all time steps, based on previously defined pumping rules, the solution $\mathbf{w}$ of the corresponding nonlinear system of equations $\mathbf{f}(\mathbf{w})=\mathbf{0}$, written in (8), proved to be highly dependent on the starting guess (the column vector $\mathbf{w 0}$ ).

We present in tables 1 and 2, all speed factor values $\left\{\omega_{21}(\mathrm{t}), \omega_{22}(\mathrm{t}), \omega_{23}(\mathrm{t})\right\}$, where $t=1 \div 24$, computed for the classical pumping algorithm, as well as for the improved pumping algorithm, for both formulations: DDA and PDA.

Table 1. Speed factors for the classical pumping algorithm.

\begin{tabular}{|c||c|c|c||c|c|c|}
\hline \multirow{2}{*}{\multicolumn{1}{|c||}{$t$}} & \multicolumn{3}{c||}{ DDA formulation } & \multicolumn{3}{c|}{ PDA formulation } \\
\cline { 2 - 7 } & $\omega_{21}$ & $\omega_{22}$ & $\omega_{23}$ & $\omega_{21}$ & $\omega_{22}$ & $\omega_{23}$ \\
\hline \hline 1 & 0.857 & 0 & 0 & 0.844 & 0 & 0 \\
\hline 2 & 0.857 & 0 & 0 & 0.844 & 0 & 0 \\
\hline 3 & 0.857 & 0 & 0 & 0.844 & 0 & 0 \\
\hline 4 & 0.857 & 0 & 0 & 0.844 & 0 & 0 \\
\hline 5 & 0.962 & 0 & 0 & 0.946 & 0 & 0 \\
\hline 6 & 1 & 0.793 & 0 & 1 & 0.769 & 0 \\
\hline 7 & 1 & 0.999 & 0 & 1 & 0.994 & 0 \\
\hline 8 & 1 & 1 & 0.980 & 1 & 1 & 0.913 \\
\hline 9 & 1 & 1 & 1.136 & 1 & 1 & 1.059 \\
\hline 10 & 1 & 1 & 0.980 & 1 & 1 & 0.913 \\
\hline 11 & 1 & 1 & 1.136 & 1 & 1 & 1.059 \\
\hline 12 & 1 & 1 & 1.136 & 1 & 1 & 1.059 \\
\hline 13 & 1 & 1 & 1.058 & 1 & 1 & 1.011 \\
\hline 14 & 1 & 1 & 0.980 & 1 & 1 & 0.913 \\
\hline 15 & 1 & 1 & 0.980 & 1 & 1 & 0.913 \\
\hline 16 & 1 & 1 & 1.136 & 1 & 1 & 1.059 \\
\hline 17 & 1 & 1 & 0.980 & 1 & 1 & 0.913 \\
\hline 18 & 1 & 1 & 1.136 & 1 & 1 & 1.059 \\
\hline 19 & 1 & 1 & 0.980 & 1 & 1 & 0.913 \\
\hline 20 & 1 & 1 & 0.847 & 1 & 1 & 0.813 \\
\hline 21 & 1 & 0.975 & 0 & 1 & 0.947 & 0 \\
\hline 22 & 1 & 0.793 & 0 & 1 & 0.769 & 0 \\
\hline 23 & 0.962 & 0 & 0 & 0.946 & 0 & 0 \\
\hline 24 & 0.962 & 0 & 0 & 0.946 & 0 & 0 \\
\hline & & & & & & \\
\hline
\end{tabular}

Table 2. Speed factors for the improved pumping algorithm.

\begin{tabular}{|c|c|c|c|c|c|c|}
\hline \multirow{2}{*}{$t$} & \multicolumn{3}{|c|}{ DDA formulation } & \multicolumn{3}{|c|}{ PDA formulation } \\
\hline & $\omega_{21}$ & $\omega_{22}$ & $\omega_{23}$ & $\omega_{21}$ & $\omega_{22}$ & $\omega_{23}$ \\
\hline 1 & 0.857 & 0 & 0 & 0.844 & 0 & 0 \\
\hline 2 & 0.857 & 0 & 0 & 0.844 & 0 & 0 \\
\hline 3 & 0.857 & 0 & 0 & 0.844 & 0 & 0 \\
\hline 4 & 0.857 & 0 & 0 & 0.844 & 0 & 0 \\
\hline 5 & 0.962 & 0 & 0 & 0.946 & 0 & 0 \\
\hline 6 & 0.868 & 0.868 & 0 & 0.859 & 0.859 & 0 \\
\hline 7 & 1.005 & 1.005 & 0 & 0.999 & 0.999 & 0 \\
\hline 8 & 0.986 & 0.986 & 0.986 & 0.972 & 0.972 & 0.972 \\
\hline 9 & 1.026 & 1.026 & 1.026 & 1.021 & 1.021 & 1.021 \\
\hline 10 & 0.986 & 0.986 & 0.986 & 0.972 & 0.972 & 0.972 \\
\hline 11 & 1.026 & 1.026 & 1.026 & 1.021 & 1.021 & 1.021 \\
\hline 12 & 1.026 & 1.026 & 1.026 & 1.021 & 1.021 & 1.021 \\
\hline 13 & 1.017 & 1.017 & 1.017 & 1.006 & 1.006 & 1.006 \\
\hline 14 & 0.986 & 0.986 & 0.986 & 0.972 & 0.972 & 0.972 \\
\hline 15 & 0.986 & 0.986 & 0.986 & 0.972 & 0.972 & 0.972 \\
\hline 16 & 1.026 & 1.026 & 1.026 & 1.021 & 1.021 & 1.021 \\
\hline 17 & 0.986 & 0.986 & 0.986 & 0.972 & 0.972 & 0.972 \\
\hline 18 & 1.026 & 1.026 & 1.026 & 1.021 & 1.021 & 1.021 \\
\hline 19 & 0.986 & 0.986 & 0.986 & 0.972 & 0.972 & 0.972 \\
\hline 20 & 0.953 & 0.953 & 0.953 & 0.936 & 0.936 & 0.936 \\
\hline 21 & 0.996 & 0.996 & 0 & 0.973 & 0.973 & 0 \\
\hline 22 & 0.868 & 0.868 & 0 & 0.859 & 0.859 & 0 \\
\hline 23 & 0.962 & 0 & 0 & 0.946 & 0 & 0 \\
\hline 24 & 0.962 & 0 & 0 & 0.946 & 0 & 0 \\
\hline
\end{tabular}

To exemplify results, we present in table 3 the computed results for the PDA formulation, for $c_{q}(t)=1.4$ at time $t=13$, for both classical and improved algorithms. On the left columns of table 3 , the 43 physical variables $\left(Q\right.$ in $\left[\mathrm{m}^{3} / \mathrm{s}\right], H$ in $[\mathrm{m}]$, and $\left.\omega\right)$ are doubled by their correspondent as $\mathbf{w}$-component in (8). The starting guess vector w0 is provided, near the final solution w, for each algorithm: due to convergence difficulties, initial values of nodal heads $H_{7} \div H_{18}$ differ from one pumping algorithm to another. Pumping head values in [m], available demands $Q_{a}$ in $\left[\mathrm{m}^{3} / \mathrm{s}\right]$, pressure head deficit $\left(H_{18}-H_{r e f}\right)$ in [m], and total demand shortage in $\left[\mathrm{m}^{3} / \mathrm{s}\right]$ are also included at the end of table 3 .

As one can notice in table 3 , the available nodal heads at the consumers with ID $=\{11 ; 12 ; 14 ; 16\}$ are greater than the reference head $\left(H_{r e f}=40 \mathrm{~m}\right)$, meaning that the pressure - demand relationship (4) fits the DDA case, so for those 4 consumers, the available demand $Q_{a}(t)$ equals the requested demand $Q_{r}(t)$. At the last consumer, with ID $=18$, there is a pressure deficit, the available nodal head being less than $40 \mathrm{~m}$ (here, a pressure drop of $0.75 \mathrm{mWC}$ is obtained for the classical algorithm, and a drop of $0.45 \mathrm{mWC}$ is obtained for the improved algorithm). For that last consumer, the relationship (4) gives $Q_{a 18}(t)<Q_{r}(t)$. The mass balance (5) compared to the requested total demand, i.e. $5 Q_{r}(t)$, shows a small water demand shortage (the relative error with respect to the requested total demand being of $-0.19 \%$ for the classical algorithm, and $-0.11 \%$ for the improved one).

The above behaviour of the computed results and the above comments apply for all 24 time steps, and for both pumping algorithms, within the PDA formulation: thus, at any time $t$, the last consumer experiences a pressure 
deficit and a slight shortage in available demand: the maximum pressure drop (1.29 $\mathrm{mWC})$ corresponds to the classical algorithm, for $c_{q}(t)=1.32$, at $t=\{8 ; 10 ; 14 ; 15$; $17 ; 19\}$, where the relative error with respect to the requested total demand is of $-0.33 \%$; the minimal pressure drop $(0.002 \mathrm{mWC})$ is negligible and corresponds to the improved algorithm, for $c_{q}(t)=1.2$, at $t=20$.

Table 3. Computed results for PDA formulation, for $c_{q}(t)=1.4$ at $t=13$, for both classical and improved algorithms.

\begin{tabular}{|c|c|c|c|c|c|}
\hline \multirow{2}{*}{\multicolumn{2}{|c|}{$\begin{array}{c}Q \text { in }\left[\mathrm{m}^{3} / \mathrm{s}\right] \\
H \text { in }[\mathrm{m}]\end{array}$}} & \multicolumn{2}{|c|}{ (1) classical } & \multicolumn{2}{|c|}{ (2) improved } \\
\hline & & \multirow{2}{*}{$\begin{array}{c}\text { w0 } \\
0.02\end{array}$} & \multirow{2}{*}{$\begin{array}{c}\mathbf{w} \\
0.6148 \\
\end{array}$} & \multirow{2}{*}{$\begin{array}{l}\text { w0 } \\
0.02\end{array}$} & \multirow{2}{*}{$\begin{array}{c}\mathbf{w} \\
0.6153\end{array}$} \\
\hline$Q_{1}$ & $w_{1}$ & & & & \\
\hline$Q_{2}$ & $w_{2}$ & 0.02 & 0.2088 & 0.02 & 0.2051 \\
\hline$Q_{3}$ & $w_{3}$ & 0.02 & 0.2030 & 0.02 & 0.2051 \\
\hline$Q_{4}$ & $w_{4}$ & 0.02 & 0.2030 & 0.02 & 0.2051 \\
\hline$Q_{5}$ & $w_{5}$ & 0.02 & 0.2088 & 0.02 & 0.2051 \\
\hline$Q_{6}$ & $w_{6}$ & 0.02 & 0.2030 & 0.02 & 0.2051 \\
\hline$Q_{7}$ & $w_{7}$ & 0.02 & 0.2030 & 0.02 & 0.2051 \\
\hline$Q_{8}$ & $w 8$ & 0.02 & 0.6148 & 0.02 & 0.6153 \\
\hline$Q_{9}$ & $w_{9}$ & 0.02 & 0.3191 & 0.02 & 0.3193 \\
\hline$Q_{10}$ & $w_{10}$ & 0.02 & 0.1318 & 0.02 & 0.1318 \\
\hline$Q_{11}$ & $w_{11}$ & 0.02 & 0.2957 & 0.02 & 0.2961 \\
\hline$Q_{12}$ & $w_{12}$ & 0.02 & 0.0641 & 0.02 & 0.0642 \\
\hline$Q_{13}$ & $w_{13}$ & 0.02 & 0.0086 & 0.02 & 0.0086 \\
\hline$Q_{14}$ & $w_{14}$ & 0.02 & 0.1198 & 0.02 & 0.1199 \\
\hline$Q_{15}$ & $w_{15}$ & 0.02 & 0.0380 & 0.02 & 0.0382 \\
\hline$Q_{16}$ & $w_{16}$ & 0.02 & 0.1760 & 0.02 & 0.1761 \\
\hline$Q_{17}$ & $w_{17}$ & 0.02 & 0.0227 & 0.02 & 0.0228 \\
\hline$Q_{18}$ & $w_{18}$ & 0.02 & 0.0466 & 0.02 & 0.0468 \\
\hline$Q_{19}$ & $w_{19}$ & 0.02 & 0.0528 & 0.02 & 0.0529 \\
\hline$Q_{20}$ & $w_{20}$ & 0.02 & 0.0754 & 0.02 & 0.0757 \\
\hline$Q_{21}$ & $w_{21}$ & 0.02 & 0.2088 & 0.02 & 0.2051 \\
\hline$Q_{22}$ & $w_{22}$ & 0.02 & 0.2030 & 0.02 & 0.2051 \\
\hline$Q_{23}$ & $w_{23}$ & 0.02 & 0.2030 & 0.02 & 0.2051 \\
\hline $\mathrm{H}_{2}$ & $\begin{array}{l}w_{24} \\
\end{array}$ & 0 & -1.10 & $\begin{array}{l}0 \\
\end{array}$ & -1.11 \\
\hline $\mathrm{H}_{3}$ & $w_{25}$ & 55 & 50.95 & 55 & 51.29 \\
\hline$H_{4}$ & $w_{26}$ & 0 & -1.18 & 0 & -1.18 \\
\hline$H_{5}$ & $w_{27}$ & 0 & -1.17 & 0 & -1.18 \\
\hline$H_{6}$ & $w_{28}$ & 0 & -1.17 & 0 & -1.18 \\
\hline $\mathrm{H}_{7}$ & $w_{29}$ & 41 & 51.28 & 43 & 51.61 \\
\hline$H_{8}$ & $w_{30}$ & 41 & 51.26 & 43 & 51.61 \\
\hline$H_{9}$ & $w_{31}$ & 41 & 51.26 & 43 & 51.61 \\
\hline$H_{10}$ & $w_{32}$ & 41 & 47.23 & 43 & 47.56 \\
\hline$H_{11}$ & $w_{33}$ & 41 & 45.60 & 43 & 45.94 \\
\hline$H_{12}$ & $w_{34}$ & 41 & 43.34 & 43 & 43.68 \\
\hline$H_{13}$ & $w_{35}$ & 41 & 45.48 & 43 & 45.81 \\
\hline$H_{14}$ & $w_{36}$ & 41 & 43.09 & 43 & 43.41 \\
\hline$H_{15}$ & w37 & 41 & 40.93 & 43 & 41.24 \\
\hline$H_{16}$ & $w_{38}$ & 41 & 43.41 & 43 & 43.73 \\
\hline$H_{17}$ & $w_{39}$ & 41 & 41.27 & 43 & 41.58 \\
\hline$H_{18}$ & $w_{40}$ & 41 & 39.25 & 43 & 39.55 \\
\hline$\omega_{21}$ & $w_{41}$ & 1 & 1.0108 & 1 & 1.0059 \\
\hline$\omega_{22}$ & $w_{42}$ & 1 & 1 & 1 & 1.0059 \\
\hline$\omega_{23}$ & $w_{43}$ & 1 & 1 & 1 & 1.0059 \\
\hline \multicolumn{3}{|c|}{$H_{21}=H_{7}-H_{4}[\mathrm{~m}]$} & 52.46 & & 52.78 \\
\hline \multicolumn{3}{|c|}{$H_{22}=H_{8}-H_{5}[\mathrm{~m}]$} & 52.44 & & 52.78 \\
\hline \multicolumn{3}{|c|}{$H_{23}=H_{9}-H_{6}[\mathrm{~m}]$} & 52.44 & & 52.78 \\
\hline \multicolumn{3}{|c|}{$\begin{array}{c}Q_{a 11}=Q_{a 12}=Q_{a 14}= \\
Q_{a 16}=Q_{r}\left[\mathrm{~m}^{3} / \mathrm{s}\right]\end{array}$} & 0.1232 & & 0.1232 \\
\hline \multicolumn{3}{|c|}{$Q_{a 18}<Q_{r}\left[\mathrm{~m}^{3} / \mathrm{s}\right]$} & 0.1220 & & 0.1225 \\
\hline \multicolumn{3}{|c|}{$H_{18}-H_{r e f}[\mathrm{~m}]$} & -0.75 & & -0.45 \\
\hline \multicolumn{3}{|c|}{$\Sigma Q_{a}-\Sigma Q_{r}\left[\mathrm{~m}^{3} / \mathrm{s}\right]$} & -0.0012 & & -0.0007 \\
\hline
\end{tabular}

Within the DDA formulation, for all 24 time steps, and both pumping algorithms, the computed solutions produce overpressures at all 5 consumers - a maximum excess pressure at the last consumer, of $3.01 \mathrm{mWC}$, corresponds to the classical algorithm, for $c_{q}(t)=1.44$, at $t=\{9 ; 11 ; 12 ; 16 ; 18\})$. Pressure reducing valves can be used to decrease the pressure at the end-users.

We present in table 4 , the computed values of the daily energy $E$ (in $\mathrm{kWh}$ ), consumed by the pumping station, and its total cost $C$ (in $€$ ), for both DDA and PDA formulations, within each pumping algorithm: the classical one and the improved one. For comparison, we also present the results obtained by Georgescu [12], for the same WDN, with another methodology within the DDA formulation. The speed factor values from tables 1 and 2 attached to DDA, can be compared to the corresponding values from Georgescu [12].

Table 4. Daily energy $E$ (in $\mathrm{kWh}$ ) consumed by the pumping station, and its total cost $C$ (in $€$ ), for the classical pumping algorithm (denoted (1)), and the improved one (denoted (2)).

\begin{tabular}{|c|c|c|c|c|c|c|}
\hline \multirow{4}{*}{ 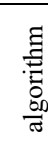 } & \multicolumn{4}{|c|}{ computed results } & \multirow{2}{*}{\multicolumn{2}{|c|}{$\frac{\text { from [12] }}{\text { DDA }}$}} \\
\hline & \multicolumn{2}{|c|}{ DDA } & \multicolumn{2}{|c|}{ PDA } & & \\
\hline & $E$ & $C$ & $E$ & $C$ & $E$ & $C$ \\
\hline & {$[\mathrm{kWh}]$} & {$[€]$} & {$[\mathrm{kWh}]$} & {$[€]$} & {$[\mathrm{kWh}]$} & {$[€]$} \\
\hline (1) & 8501 & $\overline{582}$ & 8041 & 550 & 8186 & $\overline{c 560}$ \\
\hline (2) & 8219 & 562 & 7961 & 544 & 8084 & 553 \\
\hline
\end{tabular}

Data synthesised in table 4 reveal the following: - solutions attached to PDA within both pumping algorithms ensure energy and cost savings with respect to solutions given by DDA in this paper or previously [12];

- from the energy consumption and cost points of view, the best solution is the one attached to PDA within the improved pumping algorithm - the same conclusion was drawn in previous studies on the same pumping algorithm [6, 7, 12];

- comments can arise for the fact that PDA formulation gave a pressure deficit (of less than 0.1 bar) and a slight demand shortage (of about 1 litre/second) at the most disadvantaged consumer (out of 5 consumers): it is true, but PDA is the correct formulation, while DDA is not (in real WDNs, consumers do not receive the requested demand at any available pressure). Moreover, the overpressure at the upstream consumers is smaller in PDA (less than $6 \mathrm{mWC}$ for $c_{q}(t)=1.4$ in table 3 ) than in DDA (where it reaches about $8 \mathrm{mWC}$ for $c_{q}(t)=1.4$ for both pumping algorithms);

- solutions obtained with DDA in this paper, with both pumping algorithms, gave greater energy and cost values than the corresponding solutions obtained by Georgescu [12] (relative errors vary from $1.67 \%$ to $3.88 \%$ ). The reason relies on methodologies: within this paper, the solution is computed quickly, through the nonlinear system of equations (8), but convergence depends strongly on the starting guess (for the same time step, different starting values yield different solutions, so one must choose the best solution; for some time steps, the selected computed results are convenient, while for other time steps, the selected results deviate from the expected values). The DDA methodology proposed previously 
[12] is quite accurate, but time consuming, since it involves two steps [6]: firstly, a split of the initial WDN into 2 separate hydraulic networks (meaning a change in topology), allowing to compute conveniently the unknown speed factor values and to establish speed patterns for the next step); the second step consists in restoration of the initial WDN configuration and its modelling in EPANET, based on predefined speed patterns (thus, speed factors become known input data in EPANET).

\section{Conclusions}

This paper focuses on a methodology allowing to derive the pumping schedule in Water Distribution Networks (WDN), upon a time dependent demand pattern. The selected test case is a simple WDN fed by variable speed driven pumps, previously studied [12]. Two pumping algorithms, namely a classical one and an improved one, allow selecting different pumping rules. Energy balance equations, mass balance equations and additional pumping rules form a nonlinear system of equations, which allows computing pumps speed factors. The hydraulic analysis is conducted over a period of 24 hours, with a hourly time step, within two different approaches, namely the Demand Driven Analysis (DDA) and the Pressure Driven Analysis (PDA).

The results obtained previously [12], using another DDA-based methodology, proved to be better than the results computed here with the DDA formulation. But differences are small (the maximum relative error being less than $3.9 \%$ ), so the present methodology can prevail, being more rapid. Solutions attached to PDA correspond to greater savings in terms of energy and cost than any other solution given by DDA here, or previously [12].

The methodology described in this paper is simple and rapid, but the iterative numerical method used to solve the system of equations proved to be highly dependent on the starting guess. Further work will focus on other numerical method(s), appropriate to solve the nonlinear system of equations, if possible, in a globally convergent manner.

\section{References}

1. S.-C. Georgescu, A.-M. Georgescu, R.A. Madularea, V.-F. Piraianu, A. Anton, G. Dunca, Procedia Engineering, 119, 660 (2015) https://doi. org/10.1016/j.proeng.2015.08.919

2. A.-M. Georgescu, S. Perju, S.-C. Georgescu, A. Anton, Procedia Engineering, 70, 707 (2014) https://doi.org/10.1016/j.proeng.2014.02.077

3. L. Rossman, EPANET 2 Users Manual, U.S. Environmental Protection Agency, EPA/600/R00/057 (2000) https://www.epa.gov/water-research/ epanet

4. O. Giustolisi, D.A. Savic, L. Berardi, D. Laucelli, An Excel based solution to bring water distribution network analysis closer to users, Proc. $11^{\text {th }}$ Int.
Conf. Computing and Control for the Water Industry - CCWI, 3, 805 (2011)

5. J. Muranho, A. Ferreira, J. Sousa, A. Gomes, A. Sá Marques, Procedia Engineering, 89, 632 (2014) https://doi.org/10.1016/j.proeng.2014.11.488

6. S.-C. Georgescu, A.-M. Georgescu, Hydraulic networks analysis using GNU Octave, in Romanian (Printech Press, Bucharest, 2014) https://www. researchgate.net/publication $/ 303805379$

7. S.-C. Georgescu, R. Popa, U.P.B. Sci. Bull., Series D, 72(1), 77 (2010) https://www.scientificbulletin. upb.ro/full1774.pdf

8. O. Giustolisi, T.M. Walski, J. Water Res. Pl.-ASCE, 138(4), 356 (2012) https://doi.org/10.1061/(ASCE) WR.1943-5452.0000187

9. G. Germanopoulos, Civ. Eng. Syst., 2(3), 171 (1985) https://doi.org/10.1080/02630258508970401

10. O. Giustolisi, D. Savic, Z. Kapelan, J. Hydraul. Eng., 134(5), 626 (2008) https://doi.org/10.1061/ (ASCE)0733-9429(2008)134:5(626)

11. A.-M. Georgescu, R.A. Madularea, P.-O. Ciuc, S.C. Georgescu, Decision support for a centre pivot irrigation system based on numerical modelling, HIC 2018. 13th Int. Conf. on Hydroinformatics, EPiC Series in Engineering, 3, 764 (2018) https:// doi.org/10.29007/frqh

12. S.-C. Georgescu, A.-M. Georgescu, U.P.B. Sci. Bull., Series D, 77(2), 235 (2015) https://www. scientificbulletin.upb.ro/full162 198380.pdf

13. A.-M. Georgescu, S. Perju, R.A. Madularea, S.-C. Georgescu, Energy consumption due to pipe background leakage in a district water distribution system in Bucharest, IEEE Conf. Publications, 2017 Int. Conf. on Energy and Environment CIEM (2017) https://ieeexplore.ieee.org/document/8120785/

14. O. Giustolisi, L. Berardi, D. Laucelli, D. Savic, Z. Kapelan, J. Water Res. Pl.-ASCE, 142(5), (2016) https://doi.org/10.1061/(ASCE)WR.1943-5452.0000 $\underline{583}$

15. ***, Order 41/2013 of Regulatory Authority for Energy in Romania ANRE, in Romanian, M. Of. Romania, Part I, 378 (2013)

16. J.W. Eaton, D. Bateman, S. Hauberg, R. Wehbring, GNU Octave. A high-level interactive language for numerical computations, $4^{\text {th }}$ ed. Octave 4.4.1 (2018) https://octave.org/octave.pdf

17. ***, MATLAB Documentation/ Optimization toolbox/ Systems of nonlinear equations/ fsolve (2018) https://www.mathworks.com/help/optim/ug/ fsolve.html

18. W. Press, S. Teukolsky, W. Vetterling, B. Flannery, Numerical recipes in FORTRAN. The art of scientific computing, $2^{\text {nd }}$ ed. (Cambridge University Press, 1992) 